\title{
Evaluation of the E-Learning Program Impact over Organizations in the Romanian Pharmaceutical Industry
}

\author{
Petruţa Blaga, Manuela Rozalia Gabor \\ Petru Maior University of Tirgu Mureş County, ROMANIA.
}

\begin{abstract}
This research aims to measure the impact of some initiatives within the human resources over an organization, market leader in the pharmaceutical field, the measurement of efficiency concerning the business education programs by human resources e-learning, respectively. Under the circumstances, the research carried-out allow for the application into practice of the theoretical frame of the ROI methodology (Return On Investment) of evaluating the education programs in the human resources business in five stages, suggested by J.J. Phillips. The research validates theoretical data of the model and is focused on the analysis of the gathering process of data required to apply the ROI methodology in the pharmaceutical field. Due to the complexity of this research, this paper shows only the evaluation stage of the e-learning training program impact over the organization, aspects related to measuring participants' feedback to the e-learning training program being already published by authors. Research carried-out has aimed to measure the real organizational changes as a result of training.
\end{abstract}

Key words: E-learning, Business Education, ROI methodology, Romanian Pharmaceutical Industry, Evaluation of E-learning Program.

Key Messages (Provide appropriate messages of about 35-50 words to be printed in centre box).

\section{INTRODUCTION}

Human resources represent an important and active resource of any organization that can influence directly the level of organization performance, being involved in the planning, run and increase of activity efficiency. Under the circumstances, management of organizations is trying to pay an increasingly high attention to the permanent learning process, outlining and developing a series of initiatives in the field of human resources, ${ }^{1,2}$ in order to provide the survival, development and competitional success of organizations.

The capability of organizations to accumulate and apply new knowledge represents a critical factor in achieving the new competitive standards. The continual changing diversity to which is added the need of adaptation to the external environment changes speed up the pace of evolution and learning. ${ }^{3}$ The need of survival on the market requires the organisations an increasingly higher pace. The new information and communication technologies $^{4}$ speed up the changing pace and increase the learning need that is subject to an increased information flow.

This research aims to measure the impact of some initiatives within the human resources over an organization, market leader in the pharmaceutical field, the measurement of efficiency concerning the business education programs by human resources e-learning, ${ }^{5,6,7}$ respectively. Under the circumstances, the research carried-out allow for the application into practice of the theoretical frame of
Submission Date: 19-02-2016 Revision Date : 07-04-2016 Accepted Date : 22-04-2016

DOI: 10.5530/ijper.50.4.3 Correspondence: Manuela Rozalia Gabor Nicolae lorga Street, no. 1, 540 088, TirguMureş, Mureş, County, ROMANIA. Email: rozalia.gabor@ea.upm.ro

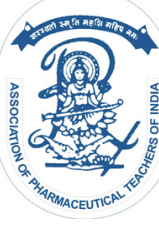

www.ijper.org 
the ROI methodology (Return On Investment) of evaluating the education programs in the human resources business in five stages, suggested by J. J. Phillips. ${ }^{8-10}$ The research validates theoretical data of the model and is focused on the analysis of the gathering process of data required to apply the ROI methodology in the pharmaceutical field. Due to the complexity of this research, this paper shows only the evaluation stage of the e-learning training program impact over the organization, aspects related to measuring participants' feedback to the e-learning training program being already published by authors. ${ }^{11}$

Most human resources managers and specialists consider that under the financial crisis, when most organizations talk about efficiency in spending their budgets and personnel restructuring, human resources investments and providing training programs can have significant results. ${ }^{12}$ Actually, most organizations has difficulties when they should decide what training programs they should invest in, how much they should invest in and if a certain initiative in the field of human resources will provide added value or not.

Therefore, human resources evaluation and taking the related measures should be present in any organization. The importance of evaluating human resources development initiatives within an organization is related to measuring investment profitability in such kind of projects. The uncertainty of measuring investment return in training programs is intensely talked about and is highly controversial. Budgets earmarked to human resources departments, competition, profitability, lack of qualified human resources has raised the importance of evaluating measurable effects of human resources training initiatives.

One of the most common measuring methodology as regards financial terms of the impact that human resources training projects have on the economic indicators of an organization is the ROI Methodology (Return on Investment), developed by Dr. Jack J. Phillips in over 30 years of research. ${ }^{8-10,13-15}$

\section{THEORETICAL AND APPLIED ASPECTS OF THE ROI METHODOLOGY}

According to Donald L. Kirkpatrick there are 4 evaluation levels of some human resources training programs,${ }^{16-21}$ feedback, learning, behavior, results.

Continuing the issues approached by D.L. Kirkpatrick, Jack J. Phillipshas emphasized an evaluation methodology of buman resources training programs in five stages, ${ }^{13-15,22}$ developing the four levels of D.L. Kirkpatrick and adding a fifth level.
- The first evaluation level, feedback, satisfaction and planned actions, measures the satisfaction degree of participants in relation to the training program.

- The second evaluation level, learning and confidence inapplicability, focuses on what participants learned at the training program.

- The third evaluation level, application and implementation on the job, requires a data collection process acknowledging that participants in the training program put their learning into effect.

- The fourth evaluation level, impact on business results impact on organization, respectively, envisages improvement of the business results as a consequence of participation in the training program.

- The fifth level, ROI, monetary benefits of the training program, that is to say how much the organization has earned expressed in financial terms as a result of program implementation, ${ }^{23}$ are compared with its costs. The evaluation cycle is never full until the fifth level is evaluated. ${ }^{24-27}$

The carried-out research aims to put into practice the theoretical frame of ROI methodology of evaluating the training programs concerning human resources, suggested by J. J. Phillips. The results of this paper aim to evaluate the impact identified in an organization after completing an e-learning training program of human resources, focusing in the same time on the way in which e-learning training determines the efficiency of human resources training programs.

\section{RESEARCH METHODOLOGY}

The educational technology used in this research has been developed at "Petru Maior" University of Tîrgu-Mureş, Romania, forthemaster's degreein QualityManagement. ${ }^{28}$ The educational program ${ }^{37,38}$ is addressed to students and managers / professionals / auditors from enterprises ${ }^{29-31}$ and it was funded by National Centre for Financing the Higher Education in Bucharest (CNFIS) in 2002. The didactical technology employs a dedicated software for asynchronous distance learning namely IBM Lotus Learning Space 3.5 Forum, which is a product intended for distance asynchronous education. ${ }^{32}$ In 2012 the IBM Lotus Learning Space 3.5 Forum was replaced with Modular Object-Oriented Dynamic Learning Envinronment (Moodle), a new didactical technology. This software package is a global development project designed to support a social framework of education used for producing Internet courses and websites. The internet address of the distance learning (e-learning) educational program is:https://mimsc.upm.ro/, https://mimsc. upm.ro/moodle/. 
The sample that is the theme of the research carried-out consists of 50 employees, of a total 200 employees and consists of personnel from the quality management department, research laboratories, production departments, distribution, divided in two groups of 25 trainees each. Their age is ranged between 25 and 60 years. The structure of trainee sample is shown in Table 1.

Through the training program required, the company aims to maintain and improve the knowledge, skills and competences of their employees relating to the organization and management of quality systems built at the company. The training program requested by the organisation was Methods of analysis and evaluation of quality.

Organisation of the business education program in two groups of trainees, with two different training systems DeSiltets, 2007), ${ }^{33}$ one based on e-learning and the other developed conventionally, is due to the option expressed by trainees and accepted by the beneficiary's management. $^{34-36,1}$

Data have been gathered by means of a questionnaire and have been processed by means of software SPSS 17.0 and Excel, using absolute and relative frequencies, simple means, average scores calculated based on mean average of data measured by means of the Likert scale, parametric and non-parametric correlations.

The variable's research are presented such as:

- 6 items in Table 2 regarding "Organisation of quality related activities";

- 7 items in Table 3 regarding "Personnel awareness";

- 10 items in Table 4 regarding the "applying the knowledge, abilities and behaviour acquired following thee-learning training program related to the quality analysis and evaluation methods had a positive effect over the following performance indicators in your activity or in your organization."

\section{PRESENTATION OF THE RESEARCH RESULTS}

As regards the organisation it helps as changing of behaviour on the job acquired following the human resources training ${ }^{37}$ in order to reflect itself in the results achieved following processing of the questionnaire entitled Evaluation of the training program impact on the organization.

In this research we present only the results for the e-learning training. The findings concerning the comparison of the opinions both of the traditional and e-learning training are in the process of publication.

Concerning question 1 of the questionnaire, for the first group of items that measures the improvement level of potential results in various fields of organisation activity that is influenced by participation of employees in the e-learning training program, respectively a value scale from 1 to 6 has been used, "does not apply", "no change","small change", "moderate change", "significant change" and "very important change" respectively, average scores being reflected in Tables 2, 3 .

Average scores for the group of questions $1 \mathrm{~A}$ in the questionnaire is ranged between 3.74 and 4.32, the minimum value 3.74 being achieved for item $1 \mathrm{Ab}$ "Application of quality management specific methods and techniques", and maximum value of 4.32 for item 1Ad "Increase of quality level in the area of responsibility". Overall for this group of questions, respondents have noticed a restrained change at organisational level following the behavioral changes of employees in the jobs resulted after training.

Average scores for the group of questions $1 \mathrm{~B}$ in the questionnaire is ranged between 3.42 and 4.16 , minimum value 3.42 being achieved with item $1 \mathrm{Bf}$ "Personnel organization within quality", and maximum value 4.16 for item $1 \mathrm{Bc}$ "Increase of number of improvement suggestions and ideas expressed by organisation personnel".

The minimum value 3.42 represents the perception of a small organisational change related to personnel organisation in the field of quality. Except this result, the results from all the other questions represents the fact that respondents have noticed a restrained organisational change.

The second question of the questionnaire "How has the organisation benefited following your participation in the e-learning training program related to the quality analysis and evaluation methods?" the respondents were required to emphasize the organization benefits measured by: reduction of the number of non-conformities, making quality assurance activities more efficient, increase of customer satisfaction, increase of employee satisfaction, increase of sales, cost cutting, time saving etc. $68.4 \%$ of respondents have noticed as the main benefit for organization the increase of quality, followed by the increase of customer satisfaction with $52.6 \%$, increase in sales with $42.1 \%$, cost cutting with $31.6 \%$, increase of employee satisfaction with $21.1 \%$, increase in productivity with $15.8 \%$.

At question 3 of questionnaire "Reflect over specific achievements / improvements specific in the quality assurance activities and think to specific methods wherein you can convert these achievements into a monetary value."Respondents were required to convert into monetary value, the achievements they noticed in the quality assurance activities in the organization. The values estimated by them have been ranged within Lei $0-120$ 000, yearly estimated value. The average 


\begin{tabular}{|c|c|c|}
\hline \multicolumn{2}{|c|}{ Table 1: Sample structure } \\
\hline \multirow{2}{*}{ Characteristic } & Sample weight \\
\hline \multirow{2}{*}{ Age } & Male & $40 \%$ \\
\cline { 2 - 3 } & Female & $60 \%$ \\
\cline { 2 - 3 } & $25-34$ years & $36 \%$ \\
\cline { 2 - 3 } & $35-49$ years & $36 \%$ \\
\hline \multirow{4}{*}{ Educational level } & $50-64$ years & $28 \%$ \\
\cline { 2 - 3 } & Higher education & $56 \%$ \\
\cline { 2 - 3 } & Postgraduate studies & $36 \%$ \\
\hline \multirow{4}{*}{\begin{tabular}{c} 
Occupational status \\
\cline { 2 - 3 }
\end{tabular}} & Doctoral studies & $8 \%$ \\
\cline { 2 - 3 } & Higher educated employee & $8 \%$ \\
\cline { 2 - 3 } & Middle educated employee & $82 \%$ \\
\hline Organisation & Management personnel & $28 \%$ \\
\cline { 2 - 3 } position & Executive personnel & $72 \%$ \\
\hline
\end{tabular}

\section{Table 2: Mean for items 1.a-1.g, Organisation of quality related activities}

$$
\text { Question - Item }
$$

Achieved mean

1. We kindly ask you to mention the improvement level of potential results from various fields of activity of your organization affected by your participation in the e-learning training program related to the quality analysis and evaluation methods.

\section{A. ORGANISATION OF QUALITY RELATED ACTIVITIES}

\begin{tabular}{|c|c|}
\hline $\begin{array}{c}\text { a. Improvement measures in organizing the quality } \\
\text { division }\end{array}$ & 3,84 \\
\hline $\begin{array}{c}\text { b. Application of quality management specific methods } \\
\text { and techniques }\end{array}$ & 3,74 \\
\hline c. Organization of current activities & 4,00 \\
\hline d. Increase of quality level in the area of responsibility & 4,32 \\
\hline e. Improvement of functional relations among divisions & 4,16 \\
\hline f. Improvement of decision-making process & 3,89 \\
\hline
\end{tabular}

\section{Table 3: Mean for items 1.a - 1.g, Personnel Awarness}

Question - Item

\section{Achieved mean}

1. We kindly ask you to mention the improvement level of potential results from various fields of activity of your organisation affected by your participation in the e-learning training program related to the quality analysis and evaluation methods.

\section{B. PERSONNEL AWARENESS}

\begin{tabular}{|c|c|}
\hline a. Increase of training level & 4,11 \\
\hline b. Efficiency of training measures & 4,11 \\
\hline $\begin{array}{l}\text { c. Increase of number of improvement suggestions and } \\
\text { ideas expressed by organisation personnel }\end{array}$ & 4,16 \\
\hline $\begin{array}{l}\text { d. Increase of number of improvement suggestions and } \\
\text { ideas applied }\end{array}$ & 3,95 \\
\hline e. Personnel motivation & 3,79 \\
\hline f. Personnel organisation in the field of quality & 3,42 \\
\hline $\begin{array}{l}\text { g. Efficiency of measures suggested within the field of } \\
\text { quality }\end{array}$ & 3,53 \\
\hline
\end{tabular}




\section{Table 4: Mean for item 7.A - 7.M}

Question - Item

Achieved mean

7. Indicate the extent to which you feel that applying the knowledge, abilities and behaviour acquired following thee-learning training program related to the quality analysis and evaluation methods had a positive effect over the following performance indicators in your activity or in your organization.

\begin{tabular}{|c|c|}
\hline A. Number of internal faults & 3,05 \\
\hline B. Number of external faults & 3,26 \\
\hline C. Identification of fault reasons & 4,16 \\
\hline D. Elimination measures concerning fault reasons & 4,16 \\
\hline E. Cost control & 4,37 \\
\hline F. Efficiency of quality assurance activities & 4,58 \\
\hline G. Response time to customers & 4,42 \\
\hline H. Increase in sales volume & 3,74 \\
\hline I. Customer satisfaction & 4,68 \\
\hline M. Customer complaints & 4,26 \\
\hline
\end{tabular}

\begin{tabular}{|c|c|c|c|c|c|c|c|c|c|}
\hline & & 1.Af & 1.Ba & 1.Bb & 1.Bc & 1.Bd & 1.Be & 1.Bf & 1.Bg \\
\hline $\begin{array}{l}\text { 1.Af Improvement of the decision- } \\
\text { making process }\end{array}$ & $\begin{array}{l}\text { Pearson Correlation } \\
\text { Sig. (2-tailed) }\end{array}$ & 1 & $\begin{array}{r}.588^{* *} \\
.008\end{array}$ & $\begin{array}{l}.561^{*} \\
.012\end{array}$ & $\begin{array}{r}.594^{* *} \\
.007\end{array}$ & $\begin{array}{r}.743^{* *} \\
.000\end{array}$ & $\begin{array}{r}.728^{* *} \\
.000\end{array}$ & $\begin{array}{r}.736^{* *} \\
.000\end{array}$ & $\begin{array}{r}.817^{* *} \\
.000\end{array}$ \\
\hline 1.Ba Raising of training level & $\begin{array}{l}\text { Pearson Correlation } \\
\text { Sig. (2-tailed) }\end{array}$ & & 1 & $\begin{array}{r}.965^{\star *} \\
.000\end{array}$ & $\begin{array}{r}.861^{* *} \\
.000\end{array}$ & $\begin{array}{r}.831^{* *} \\
.000\end{array}$ & $\begin{array}{r}.794^{* *} \\
.000\end{array}$ & $\begin{array}{r}.717^{* *} \\
.001\end{array}$ & $\begin{array}{r}.751^{* *} \\
.000\end{array}$ \\
\hline 1.Bb Efficiency of training measures & $\begin{array}{l}\text { Pearson Correlation } \\
\text { Sig. (2-tailed) }\end{array}$ & & & 1 & $\begin{array}{r}.850^{* *} \\
.000\end{array}$ & $\begin{array}{r}.785^{\star *} \\
.000\end{array}$ & $\begin{array}{r}.805^{* *} \\
.000\end{array}$ & $\begin{array}{r}.675^{* *} \\
.002\end{array}$ & $\begin{array}{r}.711^{* *} \\
.001\end{array}$ \\
\hline $\begin{array}{l}\text { 1.Bc Increase of number of } \\
\text { improvement suggestions and ideas } \\
\text { expressed by organization personnel }\end{array}$ & $\begin{array}{l}\text { Pearson Correlation } \\
\text { Sig. (2-tailed) }\end{array}$ & & & & 1 & $\begin{array}{r}.884^{* *} \\
.000\end{array}$ & $\begin{array}{r}.748^{* *} \\
.000\end{array}$ & $\begin{array}{r}.689^{* *} \\
.001\end{array}$ & $\begin{array}{r}.731^{\star *} \\
.000\end{array}$ \\
\hline $\begin{array}{l}\text { 1.Bd Increase of number of applied } \\
\text { improvement suggestions and ideas }\end{array}$ & $\begin{array}{l}\text { Pearson Correlation } \\
\text { Sig. (2-tailed) }\end{array}$ & & & & & 1 & $\begin{array}{r}.816^{* *} \\
.000\end{array}$ & $\begin{array}{r}.769^{* *} \\
.000\end{array}$ & $\begin{array}{r}.810^{* *} \\
.000\end{array}$ \\
\hline 1.BePersonnel motivation & $\begin{array}{l}\text { Pearson Correlation } \\
\text { Sig. (2-tailed) }\end{array}$ & & & & & & 1 & $.833^{* *}$ & $\begin{array}{r}.874^{* *} \\
.000\end{array}$ \\
\hline $\begin{array}{c}\text { 1.Bf Personnel organization within } \\
\text { quality }\end{array}$ & $\begin{array}{l}\text { Pearson Correlation } \\
\text { Sig. (2-tailed) }\end{array}$ & & & & & & & 1 & $\begin{array}{r}.955^{* *} \\
.000\end{array}$ \\
\hline $\begin{array}{l}\text { 1.BgEfficiency of measures } \\
\text { suggested within quality }\end{array}$ & $\begin{array}{l}\text { Pearson Correlation } \\
\text { Sig. (2-tailed) }\end{array}$ & & & & & & & & 1 \\
\hline
\end{tabular}

**. Correlation is significant at the 0.01 level (2-tailed).

*. Correlation is significant at the 0.05 level (2-tailed).

\begin{tabular}{|c|c|c|c|c|c|c|c|c|c|c|}
\hline & & & 1.Af & 1.Ba & 1. Bb & 1.BC & 1.Bd & 1.Be & 1.Bf & 1.Bg \\
\hline $\begin{array}{l}\text { Kendall's } \\
\text { tau_b }\end{array}$ & $\begin{array}{c}\text { 1.Af Improvement of } \\
\text { the decision-making } \\
\text { process }\end{array}$ & $\begin{array}{l}\text { Correlation Coef. } \\
\text { Sig. (2-tailed) }\end{array}$ & $\begin{array}{c}1.000 \\
.\end{array}$ & $\begin{array}{l}.572^{* *} \\
.003\end{array}$ & $\begin{array}{l}.525^{* *} \\
.006\end{array}$ & $\begin{array}{l}.528^{* *} \\
.005\end{array}$ & $\begin{array}{l}.648^{*+} \\
.001\end{array}$ & $\begin{array}{l}.623^{* *} \\
.001\end{array}$ & $\begin{array}{l}.665^{* *} \\
.000\end{array}$ & $\begin{array}{l}.723^{*+1} \\
.000\end{array}$ \\
\hline $\begin{array}{l}\text { Spearman's } \\
\text { rho }\end{array}$ & $\begin{array}{c}\text { 1.Af Improvement of } \\
\text { the decision-making } \\
\text { process }\end{array}$ & $\begin{array}{l}\text { Correlation Coef. } \\
\text { Sig. (2-tailed) }\end{array}$ & 1.000 & $\begin{array}{l}.666^{*+} \\
.002\end{array}$ & $\begin{array}{l}.637^{* *} \\
.003\end{array}$ & $\begin{array}{l}.667^{* *} \\
.002\end{array}$ & $\begin{array}{l}.749^{*+} \\
.000\end{array}$ & $\begin{array}{l}.734^{* *} \\
.000\end{array}$ & $\begin{array}{l}.756^{* *} \\
.000\end{array}$ & $\begin{array}{l}.826^{* *} \\
.000\end{array}$ \\
\hline
\end{tabular}

**. Correlation is significant at the 0.01 level (2-tailed). 


\begin{tabular}{|c|c|c|c|c|c|c|c|c|}
\hline \multicolumn{3}{|c|}{ lable 1: Pearson coerilcients for items $1 . \mathrm{B}$ a - $1 . \mathrm{Bg}$} & $1 . \mathrm{Bb}$ & 1.Bc & 1.Bd & 1. Be & $1 . \mathrm{Bf}$ & $1 . \mathrm{Bg}$ \\
\hline 1.Ba Raising of training level & $\begin{array}{l}\text { Pearson Correlation } \\
\text { Sig. (2-tailed) }\end{array}$ & 1 & $\begin{array}{l}.588^{* *} \\
.008\end{array}$ & $\begin{array}{l}.561^{*} \\
.012\end{array}$ & $\begin{array}{l}.594^{* *} \\
.007\end{array}$ & $\begin{array}{l}.743^{* *} \\
.000\end{array}$ & $\begin{array}{l}.128^{* *} \\
.000\end{array}$ & $\begin{array}{l}.736 \\
.000\end{array}$ \\
\hline 1.Bb Efficiency of training measures & $\begin{array}{l}\text { Pearson Correlation } \\
\text { Sig. (2-tailed) }\end{array}$ & & 1 & $\begin{array}{l}.965^{* *} \\
.000\end{array}$ & $\begin{array}{l}.861^{* *} \\
.000\end{array}$ & $\begin{array}{l}.831^{* *} \\
.000\end{array}$ & $\begin{array}{l}.794^{* *} \\
.000\end{array}$ & $\begin{array}{l}.717^{*} \\
.001\end{array}$ \\
\hline $\begin{array}{c}\text { 1.Bc Increase of number of improvement } \\
\text { suggestions and ideas expressed by } \\
\text { organization personnel }\end{array}$ & $\begin{array}{l}\text { Pearson Correlation } \\
\text { Sig. (2-tailed) }\end{array}$ & & & 1 & $\begin{array}{l}.850^{* *} \\
.000\end{array}$ & $\begin{array}{l}.785^{* \prime} \\
.000\end{array}$ & $\begin{array}{l}.805^{* *} \\
.000\end{array}$ & $\begin{array}{l}.675 \\
.002\end{array}$ \\
\hline $\begin{array}{l}\text { 1.Bd Increase of number of applied } \\
\text { improvement suggestions and ideas }\end{array}$ & $\begin{array}{l}\text { Pearson Correlation } \\
\text { Sig. (2-tailed }\end{array}$ & & & & 1 & $\begin{array}{l}.884^{* *} \\
.000\end{array}$ & $\begin{array}{l}.748^{* *} \\
.000\end{array}$ & $\begin{array}{l}.689^{*} \\
.001\end{array}$ \\
\hline 1.Be Personnel motivation & $\begin{array}{l}\text { Pearson Correlation } \\
\text { Sig. (2-tailed }\end{array}$ & & & & & 1 & $\begin{array}{l}.816^{* *} \\
.000\end{array}$ & $\begin{array}{l}.769 \\
.000\end{array}$ \\
\hline 1.Bf Personnel organization within quality & $\begin{array}{l}\text { Pearson Correlation } \\
\text { Sig. (2-tailed) }\end{array}$ & & & & & & 1 & $\begin{array}{l}.833^{*} \\
.000\end{array}$ \\
\hline $\begin{array}{c}\text { 1.Bg Efficiency of measures suggested } \\
\text { within quality }\end{array}$ & $\begin{array}{l}\text { Pearson Correlation } \\
\text { Sig. (2-tailed) }\end{array}$ & & & & & & & 1 \\
\hline
\end{tabular}

**. Correlation is significant at the 0.01 level (2-tailed).

\begin{tabular}{|c|c|c|c|c|c|c|c|c|c|}
\hline & & & 1.Ba & 1.Bb & 1.Bc & 1.Bd & 1.Be & 1.Bf & 1.Bg \\
\hline \multirow{7}{*}{ 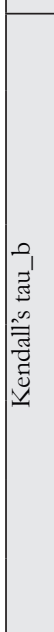 } & 1.Ba Raising of training level & \begin{tabular}{|c|}
$\begin{array}{c}\text { Correlation Coefficient } \\
\text { Sig. (2-tailed) }\end{array}$ \\
\end{tabular} & 1.000 & $\begin{array}{l}.922 \\
.000 \\
\end{array}$ & $\begin{array}{l}.780^{* *} \\
.000 \\
\end{array}$ & $\begin{array}{l}.773^{* *} \\
.000 \\
\end{array}$ & $\begin{array}{l}.754^{* *} \\
.000 \\
\end{array}$ & $\begin{array}{l}.655^{* *} \\
.001 \\
\end{array}$ & $\begin{array}{l}.707^{*+} \\
.000 \\
\end{array}$ \\
\hline & 1.Bb Efficiency of training measures & \begin{tabular}{|} 
Correlation Coefficient \\
Sig. (2-tailed)
\end{tabular} & & 1.000 & $\begin{array}{l}.799^{* *} \\
.000\end{array}$ & $\begin{array}{l}.694^{* *} \\
.000\end{array}$ & $\begin{array}{l}.759^{* *} \\
.000\end{array}$ & $\begin{array}{l}.572^{* *} \\
.002\end{array}$ & $\begin{array}{l}.623^{* *} \\
.001\end{array}$ \\
\hline & $\begin{array}{l}\text { 1.Bc Increase of number of } \\
\text { improvement suggestions and ideas } \\
\text { expressed by organization personnel }\end{array}$ & $\begin{array}{l}\text { Correlation Coefficient } \\
\text { Sig. (2-tailed) }\end{array}$ & & & 1.000 & $\begin{array}{l}.821^{* *} \\
.000\end{array}$ & $\begin{array}{l}.664^{* *} \\
.000\end{array}$ & $\begin{array}{l}.610^{* *} \\
.001\end{array}$ & $\begin{array}{l}.612^{* *} \\
.001\end{array}$ \\
\hline & $\begin{array}{l}\text { 1.Bd Increase of number of applied } \\
\text { improvement suggestions and ideas }\end{array}$ & $\begin{array}{l}\text { Correlation Coefficient } \\
\text { Sig. (2-tailed) }\end{array}$ & & & & 1.000 & $\begin{array}{l}.726^{* *} \\
.000\end{array}$ & $\begin{array}{l}.726^{* *} \\
.000\end{array}$ & $\begin{array}{l}.735^{* *} \\
.000\end{array}$ \\
\hline & 1.Be Personnel motivation & $\begin{array}{l}\text { Correlation Coefficient } \\
\text { Sig. (2-tailed) }\end{array}$ & & & & & $\begin{array}{c}1.000 \\
.\end{array}$ & $\begin{array}{l}.748^{* *} \\
.000\end{array}$ & $\begin{array}{l}.791^{* *} \\
.000\end{array}$ \\
\hline & $\begin{array}{c}\text { 1.Bf Personnel organization within } \\
\text { quality }\end{array}$ & $\begin{array}{l}\text { Correlation Coefficient } \\
\text { Sig. (2-tailed) }\end{array}$ & & & & & & 1.000 & $\begin{array}{l}.926^{*+} \\
.000\end{array}$ \\
\hline & $\begin{array}{l}\text { 1.Bg Efficiency of measures } \\
\text { suggested within quality }\end{array}$ & $\begin{array}{l}\text { Correlation Coefficient } \\
\text { Sig. (2-tailed) }\end{array}$ & & & & & & & $\begin{array}{c}1.000 \\
.\end{array}$ \\
\hline \multirow{7}{*}{ 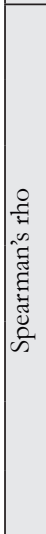 } & 1.Ba Raising of training level & $\begin{array}{l}\text { Correlation Coefficient } \\
\text { Sig. (2-tailed) }\end{array}$ & 1.000 & $\begin{array}{l}.957^{* *} \\
.000\end{array}$ & $\begin{array}{l}.884^{* *} \\
.000\end{array}$ & $\begin{array}{l}.882^{* *} \\
.000\end{array}$ & $\begin{array}{l}.849^{* *} \\
.000\end{array}$ & $\begin{array}{l}.747^{* *} \\
.000\end{array}$ & $\begin{array}{l}.771^{* *} \\
.000\end{array}$ \\
\hline & 1.Bb Efficiency of training measures & \begin{tabular}{|c|}
$\begin{array}{c}\text { Correlation Coefficient } \\
\text { Sig. (2-tailed) }\end{array}$ \\
\end{tabular} & & 1.000 & $\begin{array}{l}.895^{* *} \\
.000\end{array}$ & $\begin{array}{l}.828^{* *} \\
.000\end{array}$ & $\begin{array}{l}.855^{* *} \\
.000\end{array}$ & $\begin{array}{l}.693^{* *} \\
.001 \\
\end{array}$ & $\begin{array}{l}.721^{* *} \\
.000\end{array}$ \\
\hline & $\begin{array}{l}\text { 1.Bc Increase of number of } \\
\text { improvement suggestions and ideas } \\
\text { expressed by organization personnel }\end{array}$ & \begin{tabular}{|} 
Correlation Coefficient \\
Sig. (2-tailed)
\end{tabular} & & & 1.000 & $\begin{array}{l}.915^{*} \\
.000\end{array}$ & $\begin{array}{l}.807^{*+} \\
.000\end{array}$ & $\begin{array}{l}.747^{* *} \\
.000\end{array}$ & $\begin{array}{l}.764 * \\
.000\end{array}$ \\
\hline & $\begin{array}{l}\text { 1.Bd Increase of number of applied } \\
\text { improvement suggestions and ideas }\end{array}$ & $\begin{array}{c}\text { Correlation Coefficient } \\
\text { Sig. (2-tailed) }\end{array}$ & & & & $\begin{array}{c}1.000 \\
.\end{array}$ & $\begin{array}{l}.834^{* *} \\
.000\end{array}$ & $\begin{array}{l}.823^{* *} \\
.000\end{array}$ & $\begin{array}{l}.835^{* *} \\
.000\end{array}$ \\
\hline & 1.Be Personnel motivation & $\begin{array}{l}\text { Correlation Coefficient } \\
\text { Sig. (2-tailed) }\end{array}$ & & & & & $\begin{array}{c}1.000 \\
.\end{array}$ & $\begin{array}{l}.853^{* *} \\
.000\end{array}$ & $\begin{array}{l}.884^{* *} \\
.000\end{array}$ \\
\hline & $\begin{array}{c}\text { 1.Bf Personnel organization within } \\
\text { quality }\end{array}$ & \begin{tabular}{|c|} 
Correlation Coefficient \\
Sig. (2-tailed)
\end{tabular} & & & & & & 1.000 & $\begin{array}{l}.966^{* *} \\
.000\end{array}$ \\
\hline & $\begin{array}{l}\text { 1.Bg Efficiency of measures } \\
\text { suggested within quality }\end{array}$ & $\begin{array}{c}\text { Correlation Coefficient } \\
\text { Sig. (2-tailed) }\end{array}$ & & & & & & & 1.000 \\
\hline
\end{tabular}

of monetary values is Lei $27326.32 \%$ of respondents evaluated monetary value within Lei $0-10000$ and 36\% of respondents within Lei 10000 - 20000 .

Related to how much of monetary value estimated at question 3 has been affected by applying the knowledge and abilities achieved following the training program it resulted at question 4 of the questionnaire "What percent of above mentioned rise has been affected by applying the knowledge and abilities achieved following the e-learning training program concerning the quality analysis and evaluation methods?" an average percent of $46 \%$. This percent emphasizes that approximately half of the estimated organisation value benefits are the 


\begin{tabular}{|c|c|c|c|c|c|c|c|}
\hline & & 2.1. & 2.2 & 2.3. & 2.4. & 2.5 . & 2.6 . \\
\hline \multirow{2}{*}{ 2.1. Benefits organization $(\mathrm{BO})_{-}$increase quality } & Pearson Correlation & 1 & .121 & -.327 & .351 & .263 & -.026 \\
\hline & Sig. (2-tailed) & & .623 & .172 & .141 & .277 & .917 \\
\hline \multirow[t]{2}{*}{ 2.2. $\mathrm{BO}_{-}$increase in sales } & Pearson Correlation & & 1 & .215 & -.179 & $.809^{\star \star}$ & -.350 \\
\hline & Sig. (2-tailed) & & & .376 & .464 & .000 & .142 \\
\hline \multirow[t]{2}{*}{ 2.3. $\mathrm{BO}_{\text {_ increase productivity }}$} & Pearson Correlation & & & 1 & -.224 & .122 & .016 \\
\hline & Sig. (2-tailed) & & & & .357 & .620 & .947 \\
\hline \multirow[t]{2}{*}{ 2.4. $\mathrm{BO}_{-}$increase of employee satisfaction } & Pearson Correlation & & & & 1 & -.286 & 205 \\
\hline & Sig. (2-tailed) & & & & & .236 & .401 \\
\hline \multirow[t]{2}{*}{ 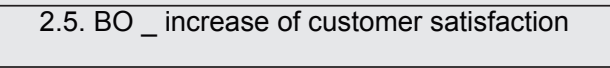 } & Pearson Correlation & & & & & 1 & $-.489^{*}$ \\
\hline & Sig. (2-tailed) & & & & & & .033 \\
\hline \multirow[t]{2}{*}{ 2.6. $\mathrm{BO}_{\text {_ }}$ cost cutting } & Pearson Correlation & & & & & & 1 \\
\hline & Sig. (2-tailed) & & & & & & \\
\hline
\end{tabular}

\begin{tabular}{|c|c|c|c|c|c|c|c|c|}
\hline & & & 2.1. & 2.2 . & 2.3. & 2.4 . & 2.5. & 2.6. \\
\hline \multirow{6}{*}{ 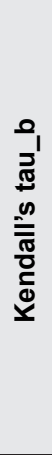 } & $2.1 \mathrm{BO}_{\text {_ increase quality }}$ & $\begin{array}{l}\text { Correlation Coef. } \\
\text { Sig. (2-tailed) }\end{array}$ & 1.000 & $\begin{array}{l}.121 \\
.609\end{array}$ & $\begin{array}{l}-.327 \\
.166\end{array}$ & $\begin{array}{l}.351 \\
.137\end{array}$ & $\begin{array}{l}.263 \\
.265\end{array}$ & $\begin{array}{l}-.026 \\
.913\end{array}$ \\
\hline & 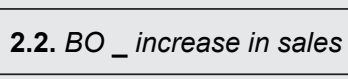 & $\begin{array}{l}\text { Correlation Coef. } \\
\text { Sig. (2-tailed) }\end{array}$ & & 1.000 & $\begin{array}{l}.215 \\
.361 \\
\end{array}$ & $\begin{array}{c}-.179 \\
.448 \\
\end{array}$ & $\begin{array}{l}.809^{* *} \\
.001\end{array}$ & $\begin{array}{l}-.350 \\
.138 \\
\end{array}$ \\
\hline & $\begin{array}{l}\text { 2.3. } \mathrm{BO} \text { _increase } \\
\text { productivity }\end{array}$ & $\begin{array}{l}\text { Correlation Coef. } \\
\text { Sig. (2-tailed) }\end{array}$ & & & $\begin{array}{c}1.000 \\
.\end{array}$ & $\begin{array}{l}-.224 \\
.343\end{array}$ & $\begin{array}{l}.122 \\
.606\end{array}$ & $\begin{array}{l}.016 \\
.945\end{array}$ \\
\hline & $\begin{array}{l}\text { 2.4. } \mathrm{BO}_{\text {e }} \text { increase of } \\
\text { employee satisfaction }\end{array}$ & $\begin{array}{l}\text { Correlation Coef. } \\
\text { Sig. (2-tailed) }\end{array}$ & & & & 1.000 & $\begin{array}{c}-.286 \\
.225\end{array}$ & $\begin{array}{l}.205 \\
.385\end{array}$ \\
\hline & $\begin{array}{l}\text { 2.5. } B_{\text {con_increase of }} \\
\text { customer satisfaction }\end{array}$ & $\begin{array}{l}\text { Correlation Coef. } \\
\text { Sig. (2-tailed) }\end{array}$ & & & & & 1.000 & $\begin{array}{r}-.489 \\
.038\end{array}$ \\
\hline & 2.6. BO_cost cutting & $\begin{array}{l}\text { Correlation Coef. } \\
\text { Sig. (2-tailed) }\end{array}$ & & & & & & 1.000 \\
\hline \multirow{6}{*}{ 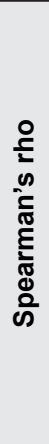 } & $2.1 \mathrm{BO}_{\text {_ increase quality }}$ & $\begin{array}{l}\text { Correlation Coef. } \\
\text { Sig. (2-tailed) }\end{array}$ & 1.000 & $\begin{array}{l}.121 \\
.623\end{array}$ & $\begin{array}{l}-.327 \\
.172\end{array}$ & $\begin{array}{l}.351 \\
.141\end{array}$ & $\begin{array}{l}.263 \\
.277\end{array}$ & $\begin{array}{l}-.026 \\
.917\end{array}$ \\
\hline & 2.2. $\mathrm{BO}_{\text {_ }}$ increase in sales & $\begin{array}{l}\text { Correlation Coef. } \\
\text { Sig. (2-tailed) }\end{array}$ & & 1.000 & $\begin{array}{l}.215 \\
.376\end{array}$ & $\begin{array}{l}-.179 \\
.464\end{array}$ & $\begin{array}{l}.809^{* *} \\
.000\end{array}$ & $\begin{array}{l}-.350 \\
.142\end{array}$ \\
\hline & $\begin{array}{l}\text { 2.3. BO_increase } \\
\text { productivity }\end{array}$ & $\begin{array}{l}\text { Correlation Coef. } \\
\text { Sig. (2-tailed) }\end{array}$ & & & $\begin{array}{c}1.000 \\
.\end{array}$ & $\begin{array}{l}-.224 \\
.357\end{array}$ & $\begin{array}{l}.122 \\
.620\end{array}$ & $\begin{array}{l}.016 \\
.947\end{array}$ \\
\hline & $\begin{array}{l}\text { 2.4. } \mathrm{BO}_{-} \text {increase of } \\
\text { employee satisfaction }\end{array}$ & $\begin{array}{l}\text { Correlation Coef. } \\
\text { Sig. (2-tailed) }\end{array}$ & & & & 1.000 & $\begin{array}{l}-.286 \\
.236\end{array}$ & $\begin{array}{l}.205 \\
.401\end{array}$ \\
\hline & $\begin{array}{l}\text { 2.5. } B O_{\text {_ }} \text { increase of } \\
\text { customer satisfaction }\end{array}$ & $\begin{array}{l}\text { Correlation Coef. } \\
\text { Sig. (2-tailed) }\end{array}$ & & & & & 1.000 & $\begin{array}{r}-.489 \\
.033\end{array}$ \\
\hline & 2.6. $B O O_{-}$cost cutting & $\begin{array}{l}\text { Correlation Coef. } \\
\text { Sig. (2-tailed) }\end{array}$ & & & & & & 1.000 \\
\hline
\end{tabular}

result of applying the knowledge and abilities acquired following training.

Related to the safety of response to question 4, respondents estimate at question 5 "How safe are you about the above mentioned estimations?" from the questionnaire that it is safe in a proportion of $72 \%$.

At question 6 in the questionnaire "Do you think that this e-learning training program related to the quality analysis and evaluation methods has represented a good investment for your organisation?”, from the total sample, $73.7 \%$ feel that training of organization human resources represents to a high extent a good investment for the organization and only $26.3 \%$ feel that training to a low extent as being a good investment for the organization.

It is worthy of note that though this question has the alternative "Not at all" as a response, none respondent has chosen it.

Concerning question 7 of the questionnaire, importance that acquiring knowledge and abilities in quality assurance achieved by employees following training, had over the organization performance indicators, respectively a value scale from 1 to 6 has been used "not applicable", "applies but has no effect", "low effect", "restrained effect", "important effect" and "very important effect" 


\begin{tabular}{|c|c|c|c|c|c|c|c|c|c|c|c|}
\hline & & 7.A & 7.B & 7.C & 7.D & 7.E & 7.F & 7.G & 7.H & 7.I. & 7.M \\
\hline \multicolumn{12}{|c|}{ Applying knowledge had positive influence on: } \\
\hline \multirow{2}{*}{ 7.A Number of internal faults } & Pearson Correlation & 1 & $.945^{\star *}$ & $.736^{\star *}$ & $.543^{*}$ & .326 & .399 & .405 & $.489^{*}$ & $.469^{*}$ & .453 \\
\hline & Sig. (2-tailed) & & .000 & .000 & .016 & .174 & .091 & .085 & .034 & .043 & .052 \\
\hline \multirow{2}{*}{ 7.B Number of external faults } & Pearson Correlation & & 1 & $.787^{* \star}$ & $636^{* \star}$ & .454 & $.501^{*}$ & $479^{*}$ & $.565^{*}$ & $554^{*}$ & $488^{*}$ \\
\hline & Sig. (2-tailed) & & & .000 & .003 & .051 & .029 & .038 & .012 & .014 & .034 \\
\hline \multirow{2}{*}{$\begin{array}{l}\text { 7.C Identification of fault } \\
\text { reasons }\end{array}$} & Pearson Correlation & & & 1 & $.740^{* *}$ & $.557^{*}$ & $.505^{*}$ & .324 & $.471^{*}$ & .347 & .366 \\
\hline & Sig. (2-tailed) & & & & .000 & .013 & .028 & 176 & .042 & .145 & .123 \\
\hline \multirow{2}{*}{$\begin{array}{l}\text { 7.D Elimination measures of } \\
\text { fault reasons }\end{array}$} & Pearson Correlation & & & & 1 & $.555^{*}$ & $.783^{* *}$ & .443 & $.721^{\star *}$ & .402 & .164 \\
\hline & Sig. (2-tailed) & & & & & .014 & .000 & .058 & .000 & .088 & .502 \\
\hline \multirow[t]{2}{*}{ 7.E Cost cutting } & Pearson Correlation & & & & & 1 & $.504^{*}$ & .151 & $.629^{* *}$ & .324 & .407 \\
\hline & Sig. (2-tailed) & & & & & & .028 & .538 & .004 & .176 & .084 \\
\hline \multirow{2}{*}{$\begin{array}{l}\text { 7.F Efficiency of quality } \\
\text { assurance activities }\end{array}$} & Pearson Correlation & & & & & & 1 & $.617^{\star *}$ & $.658^{\star *}$ & $.522^{*}$ & .119 \\
\hline & Sig. (2-tailed) & & & & & & & .005 & .002 & .022 & .626 \\
\hline \multirow{2}{*}{$\begin{array}{c}\text { 7.G Response time to } \\
\text { customers }\end{array}$} & Pearson Correlation & & & & & & & 1 & $.674^{\star \star}$ & $.829^{\star *}$ & .397 \\
\hline & Sig. (2-tailed) & & & & & & & & .002 & .000 & .092 \\
\hline \multirow[t]{2}{*}{ 7.H Increase in sales volume } & Pearson Correlation & & & & & & & & 1 & $.678^{* *}$ & $.491^{*}$ \\
\hline & Sig. (2-tailed) & & & & & & & & & .001 & .033 \\
\hline \multirow[t]{2}{*}{ 7.I. Customer satisfaction } & Pearson Correlation & & & & & & & & & 1 & $.654^{\star *}$ \\
\hline & Sig. (2-tailed) & & & & & & & & & & .002 \\
\hline \multirow[t]{2}{*}{ 7.M Customer complaints } & Pearson Correlation & & & & & & & & & & 1 \\
\hline & Sig. (2-tailed) & & & & & & & & & & \\
\hline
\end{tabular}

**. Correlation is significant at the 0.01 level (2-tailed).

*. Correlation is significant at the 0.05 level (2-tailed).

\begin{tabular}{|c|c|c|c|c|c|c|c|c|c|c|c|}
\hline & & 7.A & 7.B & 7.C & 7.D & 7.E & $7 . F$ & $7 . G$ & 7.H & 7.I. & 7.M \\
\hline \multicolumn{12}{|c|}{ Applying knowledge had positive influence on: } \\
\hline 7.A number of internal faults & $\begin{array}{l}\text { Correlation Coef. } \\
\text { Sig. (2-tailed) }\end{array}$ & $\begin{array}{c}1.000 \\
.\end{array}$ & $\begin{array}{l}.860^{* *} \\
.000\end{array}$ & $\begin{array}{l}.572^{* *} \\
.003\end{array}$ & $\begin{array}{l}.427^{*} \\
.024\end{array}$ & $\begin{array}{l}.252 \\
.183\end{array}$ & $\begin{array}{l}.297 \\
.123\end{array}$ & $\begin{array}{l}.390^{*} \\
.040\end{array}$ & $\begin{array}{l}.406^{*} \\
.032\end{array}$ & $\begin{array}{l}.385^{*} \\
.045\end{array}$ & $\begin{array}{l}.373^{*} \\
.049\end{array}$ \\
\hline 7.B Number of external faults & $\begin{array}{l}\text { Correlation Coef. } \\
\text { Sig. (2-tailed) }\end{array}$ & & 1.000 & $\begin{array}{l}.623^{* *} \\
.001\end{array}$ & $\begin{array}{l}.539^{* *} \\
.005\end{array}$ & $\begin{array}{l}.355 \\
.062\end{array}$ & $\begin{array}{l}.419^{*} \\
.030\end{array}$ & $\begin{array}{l}.460^{*} \\
.016\end{array}$ & $\begin{array}{l}.475^{*} \\
.013\end{array}$ & $\begin{array}{l}.449^{*} \\
.020\end{array}$ & $\begin{array}{l}.371 \\
.051\end{array}$ \\
\hline 7.C Identification of fault reasons & $\begin{array}{l}\text { Correlation Coef. } \\
\text { Sig. (2-tailed) }\end{array}$ & & & 1.000 & $\begin{array}{l}.652^{* *} \\
.001\end{array}$ & $\begin{array}{l}.428^{*} \\
.027\end{array}$ & $\begin{array}{l}.368 \\
.062\end{array}$ & $\begin{array}{l}.331 \\
.089\end{array}$ & $\begin{array}{l}.370 \\
.056\end{array}$ & $\begin{array}{l}.301 \\
.126\end{array}$ & $\begin{array}{l}.307 \\
.113\end{array}$ \\
\hline \multirow{2}{*}{$\begin{array}{c}\text { 7.D elimination measures of fault } \\
\text { reasons }\end{array}$} & Correlation Coef. & & & & 1.000 & $.496^{* *}$ & $.662^{* *}$ & $.439^{*}$ & $.660^{* *}$ & $.449^{*}$ & .193 \\
\hline & Sig. (2-tailed) & & & & & .009 & .001 & .022 & .001 & .021 & .312 \\
\hline 7.E cost cutting & $\begin{array}{l}\text { Correlation Coef. } \\
\text { Sig. (2-tailed) }\end{array}$ & & & & & 1.000 & $\begin{array}{l}.581^{* *} \\
.003\end{array}$ & $\begin{array}{l}.230 \\
.230\end{array}$ & $\begin{array}{l}.546^{* *} \\
.004\end{array}$ & $\begin{array}{l}.383^{*} \\
.048\end{array}$ & $\begin{array}{l}.471^{*} \\
.014\end{array}$ \\
\hline \multirow{2}{*}{$\begin{array}{l}\text { 7.F efficiency of quality } \\
\text { assurance activities }\end{array}$} & Correlation Coef. & & & & & & 1.000 & $.597^{* *}$ & $.625^{* *}$ & $.595^{* *}$ & .267 \\
\hline & Sig. (2-tailed) & & & & & & 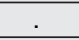 & .002 & .001 & .003 & .171 \\
\hline \multirow[t]{2}{*}{ 7.G response time to customers } & Correlation Coef. & & & & & & & 1.000 & $.619^{* *}$ & $.806^{* *}$ & .355 \\
\hline & Sig. (2-tailed) & & & & & & & 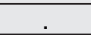 & .001 & .000 & .064 \\
\hline \multirow[t]{2}{*}{ 7.H increase in sales volume } & Correlation Coef. & & & & & & & & 1.000 & $.625^{* *}$ & $.450^{*}$ \\
\hline & Sig. (2-tailed) & & & & & & & & & .001 & .019 \\
\hline 7.I. Customer satisfaction & $\begin{array}{l}\text { Correlation Coef. } \\
\text { Sig. (2-tailed) }\end{array}$ & & & & & & & & & 1.000 & $\begin{array}{l}.556 \\
.004\end{array}$ \\
\hline 7.M Customer complaints & $\begin{array}{l}\text { Correlation Coef. } \\
\text { Sig. (2-tailed) }\end{array}$ & & & & & & & & & & $\begin{array}{c}1.000 \\
.\end{array}$ \\
\hline
\end{tabular}

**. Correlation is significant at the 0.01 level (2-tailed).

*. Correlation is significant at the 0.05 level (2-tailed).

respectively, achieving average scores for the response version illustrated in Table 4.

It is worthy of note that respondents have noticed:

- A small change for indicators: Number of internal faults - score 3.05; Number of external faults score 3.26;

- A restrained change for indicators: Identification of fault reasons - score 4.16; Elimination measures for fault reasons - score 4.16; Cost control - score 4.37; Response time to customers - score 4.42; Increase in sales volume - score 3.74; Customer complaints - score 4.26;
- A major change for indicators: Customer satisfaction - score 4.68; Efficiency of quality assurance activitiesscore 4.58 .

At question 8 in the questionnaire "What additional benefits have resulted from this e-learning training program related to the quality analysis and evaluation methods (materials / organization / job satisfaction / etc.)?" none of the respondents has answered that following training and applying the knowledge and abilities on the job could get material benefits. Only $26.3 \%$ of respondents have answered that they could get benefits concerning activity organization following training and a 


\begin{tabular}{|c|c|c|c|c|c|c|c|c|c|c|c|}
\hline Table 13: Spearman coefficients for items 7.A - 7.M & & 7.A & 7.B & 7.C & 7.D & 7.E & 7.F & 7.G & 7.H & 7.I. & 7.M \\
\hline \multicolumn{12}{|c|}{ Applying knowledge had positive influence on: } \\
\hline 7.A number of internal faults & $\begin{array}{l}\text { Correlation Coef. } \\
\text { Sig. (2-tailed) }\end{array}$ & 1.000 & $\begin{array}{l}.926^{* *} \\
.000\end{array}$ & $\begin{array}{l}.682^{* \star} \\
.001\end{array}$ & $\begin{array}{l}.498^{*} \\
.030\end{array}$ & $\begin{array}{l}.194 \\
.426\end{array}$ & $\begin{array}{l}.307 \\
.201\end{array}$ & $\begin{array}{l}.481^{*} \\
.037\end{array}$ & $\begin{array}{l}.412 \\
.080\end{array}$ & $\begin{array}{l}.425 \\
.070\end{array}$ & $\begin{array}{l}.363 \\
.126\end{array}$ \\
\hline 7.B Number of external faults & $\begin{array}{l}\text { Correlation Coef. } \\
\text { Sig. (2-tailed) }\end{array}$ & & 1.000 & $\begin{array}{l}.724^{* *} \\
.000\end{array}$ & $\begin{array}{l}.612^{* *} \\
.005\end{array}$ & $\begin{array}{l}.371 \\
.118\end{array}$ & $\begin{array}{l}.462^{*} \\
.046\end{array}$ & $\begin{array}{l}.553^{*} \\
.014\end{array}$ & $\begin{array}{l}.542^{*} \\
.016\end{array}$ & $\begin{array}{l}.514^{*} \\
.024\end{array}$ & $\begin{array}{l}.444 \\
.057\end{array}$ \\
\hline 7.C Identification of fault reasons & $\begin{array}{l}\text { Correlation Coef. } \\
\text { Sig. (2-tailed) }\end{array}$ & & & 1.000 & $\begin{array}{l}.742^{* *} \\
.000\end{array}$ & $\begin{array}{l}.513^{*} \\
.025\end{array}$ & $\begin{array}{l}.425 \\
.070\end{array}$ & $\begin{array}{l}.401 \\
.089\end{array}$ & $\begin{array}{l}.455 \\
.050\end{array}$ & $\begin{array}{l}.374 \\
.114\end{array}$ & $\begin{array}{l}.397 \\
.092\end{array}$ \\
\hline \multirow{2}{*}{$\begin{array}{l}\text { 7.D elimination measures of fault } \\
\text { reasons }\end{array}$} & Correlation Coef. & & & & 1.000 & $.564^{*}$ & $.747^{* *}$ & $.534^{*}$ & $.759^{* *}$ & $.544^{*}$ & .263 \\
\hline & Sig. (2-tailed) & & & & & .012 & .000 & .019 & .000 & .016 & .276 \\
\hline 7.E cost cutting & $\begin{array}{l}\text { Correlation Coef. } \\
\text { Sig. (2-tailed) }\end{array}$ & & & & & 1.000 & $\begin{array}{l}.633^{\star *} \\
.004\end{array}$ & $\begin{array}{l}.303 \\
.207\end{array}$ & $\begin{array}{l}.611^{* *} \\
.005\end{array}$ & $\begin{array}{l}.471^{*} \\
.042\end{array}$ & $\begin{array}{l}.550^{*} \\
.015\end{array}$ \\
\hline \multirow{2}{*}{$\begin{array}{l}\text { 7.F efficiency of quality assurance } \\
\text { activities }\end{array}$} & Correlation Coef. & & & & & & 1.000 & $.736^{\star *}$ & $.747^{* *}$ & $.728^{* *}$ & .345 \\
\hline & Sig. (2-tailed) & & & & & & . & .000 & .000 & .000 & .148 \\
\hline \multirow[t]{2}{*}{ 7.G response time to customers } & Correlation Coef. & & & & & & & 1.000 & $.694^{* *}$ & $.886^{* *}$ & .428 \\
\hline & Sig. (2-tailed) & & & & & & & & .001 & .000 & .067 \\
\hline \multirow[t]{2}{*}{ 7.H increase in sales volume } & Correlation Coef. & & & & & & & & 1.000 & $.711^{* *}$ & $.540^{*}$ \\
\hline & Sig. (2-tailed) & & & & & & & & & .001 & .017 \\
\hline 7.I. customer satisfaction & $\begin{array}{l}\text { Correlation Coef. } \\
\text { Sig. (2-tailed) }\end{array}$ & & & & & & & & & 1.000 & $\begin{array}{l}.623^{* *} \\
.004\end{array}$ \\
\hline \multirow[t]{2}{*}{ 7.M customer complaints } & Correlation Coef. & & & & & & & & & & 1.000 \\
\hline & Sig. (2-tailed) & & & & & & & & & & \\
\hline
\end{tabular}

percentage of $36.8 \%$ have felt that they could get job satisfaction.

It may conclude that financial support of the e-learning training program by the organization for its employees is not perceived as a material benefit by trainees, but it is rather a commitment.

In case of the questionnaire entitled Impact evaluation of the training program on organization, in order to fill in the results concerning the training impact over organization, achieved through horizontal statistical analysis, a vertical statistical analysis will be also applied.

A first set of correlations has been achieved reviewing question 1 "We kindly ask you to mention the improvement level of potential results from various fields of activity of your organization in conjunction with your participation in the e-learning training program related to the quality analysis and evaluation methods" on the two groups of items $1 \mathrm{~A}$ and $1 \mathrm{~B}$. Of the group of items 1A Organization of quality related activities, in order to carry-out correlations, item 1 Af "Improvement of decisional process" will be taken into consideration thanks to the major impact and direct and causal connection with the response versions achieved.

Consequently, correlation between this item 1Af and the response versions of the group of items $1 \mathrm{~B}$ personnel awareness will be tested by means of parametric and non-parametric correlation coefficients. Results will be shown in tables related to each correlation.

As a result of data processing it is noticed that among these items, the lowest values of correlation coefficients are at least moderate and increase to high intensity correlations, results that acknowledge training and training quality impact, as well as the immediate application of knowledge and abilities acquired by respondents at the job, on improving the decision-making process within the organization.

Following the positive values for correlations between item 1Af "Improvement of the decision-making process" and items $1 \mathrm{~B}$ related to a direct correlation, it can be stated that they reveal a mutual effect among these items. Hereinafter these results are detailed. (Table 5 and 6):

- Moderate value direct correlations that are statistically significant are achieved between items: $1 \mathrm{Af}$ with 1Ba,"Raising of training level" (Pearson coefficients - 0.588; Kendall - 0.572; Spearman - 0.666); $1 \mathrm{Af}$ with $1 \mathrm{Bb}$ "Efficiency of training measures" (Pearson coefficients - 0.561; Kendall - 0.525; Spearman 0.637); $1 \mathrm{Af}$ with $1 \mathrm{Bc}$ "Increase of number of improvement suggestions andideas expressed by organization personnel" (Pearson coefficients - 0.594; Kendall - 0.528; Spearman - 0.667);

- High intensity direct correlations that are statistically significant are achieved between items: $1 \mathrm{Af}$ with 1Bd "Increase of number of applied improvement suggestions and ideas" (Pearson coefficients - 0.743; Kendall - 0.648; Spearman - 0.749); 1Af with 1Be "Personnel motivation" (Pearson coefficients -0.728 ; Kendall - 0.683; Spearman - 0.734); 1Af with 1Bf "Personnel organization within quality" (Pearson coefficients - 0.736; Kendall - 0.665; Spearman - 0.756); $1 \mathrm{Af}$ with $1 \mathrm{Bg}$ "Efficiency of measures suggested within 
quality" (Pearson coefficient - 0.817; Kendall coefficient - 0.723; Spearman coefficient- 0.826).

Taking into consideration that there were both management personnel representatives and executive personnel representatives within the sample, correlations between response versions related to items $1 \mathrm{~B}$ Personnel awareness can be tested. (Tables 7 and 8):

- Moderate value direct correlations that are statistically significant are achieved for items: $1 \mathrm{Ba}$ with $1 \mathrm{~B}$ "Increase of training level" (Pearson coefficient - 0.717; Kendall coefficient -0.655 ; Spearman coefficient $0.747) ; 1 \mathrm{Bb}$ with $1 \mathrm{Bf}$ "Efficiency of training measures" (Pearson coefficient -0.675 ; Kendall coefficient - 0.572; Spearman coefficient -0.693$)$; $1 \mathrm{Bb}$ with $1 \mathrm{Bg}$ "Increase of number of improvement suggestions and ideas expressed by organization personnel" (Pearson coefficient -0.711 ; Kendall coefficient -0.623 ; Spearman coefficient - 0.721); 1Bc with 1Bf, 'Increase of improvement suggestions and ideas expressed by organization personnel" (Pearson coefficient - 0.689; Kendall coefficient- 0.610; Spearman coefficient - 0.747); 1Bc with $1 \mathrm{Bg}$, "Increase of number of improvement suggestions and ideas expressed by organization personnel" (Pearson coefficient -0.731 ; Kendall coefficient -0.612 ; Spearman coefficient - 0.764);

- High intensity direct correlations that are statistically significant are achieved between items: 1Ba with $1 \mathrm{Bb}$,"Increase of number of applied improvement suggestions and ideas" (Pearson coefficient - 0.965; Kendall coefficient -0.922 ; Spearman coefficient -0.957 ); $1 \mathrm{Bf}$ with 1Bg,"Personnel motivation" (Pearson coefficient -0.955 ; Kendall coefficient -0.926 ; Spearman coefficient -0.966$)$; $1 \mathrm{Bc}$ with $1 \mathrm{Bd}$, "Personnel organization within quality" (Pearson coefficient - 0.884; Kendall coefficient -0.821 ; Spearman coefficient - 0.915); 1Be with 1Bg, "Efficiency of measures suggested within quality" (Pearson coefficient - 0.874; Kendall coefficient -0.791 ; Spearman coefficient -0.884 ).

These correlations calculated within the group of items 1B mean the causal importance among items and as a result the conclusion is that measuring of personnel awareness should be achieved taking into account all the variables related to the group of items concerned.

Testing the correlations among the variables related to question 2 in the questionnaire "How did the organization took advantage of your participation in the e-learning training program concerning the quality analysis and evaluation methods? We kindly ask you to identify achievements or improvements specific to activity, that you think they are driven by participation in this e-learning training program" have resulted data shown in Tables 9 and 10, respectively:
- There is a high intensity direct correlation that is statistically significant between 2.2 , "increase in sales" and 2.5,"increase of customer satisfaction" (Pearson, Kendall, Spearman coefficients- 0.809); that shows the direct relation that respondents perceive between the two variables;

- There is a reverse moderate intensity correlation that is statistically significant between 2.5 "increase of customer satisfaction" and 2.6, "cost cutting" (Pearson, Kendall, Spearman coefficients $=-0,489)$. This value shows that respondents that chose the version 2.5 "increase of customer satisfaction", did not choose 2.6, "cost cutting", and in conclusion, respondents have not perceived any connection between the two variables.

In order to evaluate the impact of human resources training program by e-learning on the organization, there will analyze the correlations among performance indicators emphasized within question 7 "Mention the extent in which you consider that applying the knowledge, abilities and behaviour acquired following the e-learning training program related to the quality analysis and evaluation methods had a positive influence on the following performance indicators in your activity or in your organization":

- There are moderate intensity direct correlations that are statistically significant between: 7,"Number of internal faults" with 7C,"Identification of fault reasons" (Pearson, Kendall coefficients - 0.572; Spearman coefficient - 0.682); 7B, "Number of external faults" with 7C,"Identification of fault reasons" (Pearson, Kendall coefficients - 0.623; Spearman coefficient - 0.724); 7C,"Identification of fault reasons" with 7D,"Elimination measures of fault reasons" (Pearson, Kendall coefficients - 0.652; Spearman coefficient $-0.742) ; 7 \mathrm{D}$,"Elimination measures of fault reasons" with 7F, "Efficiency of quality assurance activities" (Pearson, Kendall coefficients - 0.662; Spearman coefficient - 0.747); 7D, "Elimination measures of fault reasons" cu $7 \mathrm{H}$, "Increase in sales volume" (Pearson, Kendall coefficients -0.660 ; Spearman coefficient -0.534 ); 7E, "Cost cutting" with 7F ,"Efficiency of quality assurance activities" (Pearson, Kendall coefficients - 0.581; Spearman coefficient - 0.633); 7F, "Efficiency of quality assurance activities" with 7G," Response time to customers" (Pearson, Kendall coefficients - 0.597; Spearman coefficient - 0.736); 7F, "Efficiency of quality assurance activities" with 7H,"Increase in sales volume" (Pearson, Kendall coefficients - 0.625; Spearman coefficient - 0.747); 7F,"Efficiency of quality assurance activities" with 7I," Customer satisfaction" (Pearson, Kendall coefficients - 0.595; Spearman coefficient 
- 0.728); 7G ,"Response time to customers" with $7 \mathrm{H}$, "Increase of sales in volume" (Pearson, Kendall coefficients - 0.619; Spearman coefficient - 0.694).

These moderate correlations acknowledge the effect of training on organising the quality related activity, correlations that identify in a series of quality management procedures in a productive unit (Tables 11,12 and 13).

- There are high intensity direct correlations that are statistically significant between: 7A, "Number of internal faults" with7B, "Number of external faults" (Pearson, Kendall coefficients - 0.860; Spearman coefficient - 0.926); 7G, "Response time to customers" with 7I,"Customer satisfaction" (Pearson, Kendall coefficient S - 0.806; Spearman coefficient - 0.886);

\section{CONCLUSIONS AND DISCUSSION}

This research has been carried-out based on a quantitative analysis according to the research methodology suggested. The quantitative nature of data resulted from research enable performance of some analyses that emphasize complexity that e-learning and its effects involve. The strategies applied within research in order to evaluate the four stages of data collection process within the ROI methodology results in getting a high number of data and the possibility to analyze and emphasize certain specific results that are relevant for the study.

Impact evaluation, the last stage of data collection process within ROI Methodology, has assumed analysis of the fourth questionnaire Impact evaluation of the training program on organization in order to determine benefits, organizational results reflected in business, respectively following the training efforts.

Research carried-out has aimed to measure the real organizational changes as a result of e-learning training and has led to the following findings:

- Trainees has perceived a moderate change at organizational level as a result of behavioral changes on the job after training.

- Trainees have perceived as the main benefit for organization quality increase, followed by increase of customer satisfaction, increase in sales, cost cutting, increase of employees satisfaction and increase in productivity;

- Respondents have tried to convert into monetary value the achievements that have been perceived in the reviewed field of activity and have set that approximately half of the estimated value benefits of the organization is due to applying the knowledge and abilities acquired following training;
- Following training and applying the knowledge and abilities on the job, respondents have estimated that benefits as regards activity organization have been achieved after training and increase of employees satisfaction on the job;

- Most trainees feel that training of organization human resources represents to a great extent a good investment for the organization.

\section{ACNOWLEDGEMENT}

NIL.

\section{CONFLICT OF INTEREST}

The authors declare no conflict of interest

\section{REFERENCES}

1. Armstrong M. Human Resources Management: practice manual. Bucharest: CODECS; 2003. Bailey KD, Morais DB. Exploring the use of blended learning in tourism education. Journal of Teaching in Travel \& Tourism. 2005;4:23-36.

2. Armstrong M. A Handbook of Human Resource Management Practice. 10th ed. London: Kogan Page Limited; 2006.

3. Lazăr CM, Bunda RN. Knowledge-based Economy, an Appropriate Response to Organizational Change Pressures, with a View to Sustainable Development. Amfiteatru Economic. 2012;14(32):302-15.

4. Liburd J, Hjalager AM. Changing Approaches towards open education, innovation and research in tourism. Journal of Hospitality and Tourism Management. 2010;17:12-20.

5. Sigala M. The evolution of Internet pedagogy: Benefits from tourism and hospitality education. Journal of Hospitality, Leisure, Sport \& Tourism Education.2002;1:27-42.

6. Sigala M. Investigating the factors determining e-learning effectiveness in tourism and hospitality education. Journal of Hospitality and Tourism Education. 2004;16(2):11-21.

7. Sigala M. Investigating the role and impact of geovisualisation and geocollaborative portals on collaborative e-learning in tourism education. Journal of Hospitality, Leisure, Sports \& Tourism Education.2012;11(1):50-66.

8. Phillips JJ. Handbook of training evaluation and measurement methods. Houston: Gulf Publishing Company; 1991.

9. Philips JJ. ROI: The search for best practices. Training \& Development 1996;50:42-7.

10. Phillips PP, Phillips JJ. Return on investment. In: Watkins R, Leight D., editors. Handbook of Improving Performance in the Workplace, Volume 2Selecting and implementing Performance Interventions. San Francisco: John Wiley and Sons; 2010.

11. Blaga P, Gabor MR. Investigating the impact of e-learning as an alternative for business education in pharmaceutical industry in Romania by ROI methodology. Amfiteatru Economic 2014;16(37):902-12. Thomas F. The use of e-learning for the development of a sustainable tourism. [online]. 2012. Available from: URL:www.cctd.eu.

12. Phillips JJ. The return-on-investment (ROI) process: Issues and trends. Educational Technology. 1998;8:7-14.

13. Philips JJ. Return on investment in training and performance improvement programs. 2nd ed. Philadelphia: Elsevier Science \& Technology; 2003.

14. Phillips JJ. Measuring ROI: The Process, Current Issues, and Trends. [online]. 2007. Available from: URL: www.roiinstitute.net

15. Kirkpatrick DL. Techniques for evaluating training programs: Reaction. American Society for Training and Development Journal. 1959a;18:3-9.

16. Kirkpatrick DL. Techniques for evaluating training programs: Learning. American Society for Training and Development Journal. 1959b;18:21-6. 
17. Kirkpatrick DL. Techniques for evaluating training programs: Behavior. American Society for Training and Development Journal. 1960a;19:13-18.

18. Kirkpatrick DL. Techniques for evaluating training programs: Learning. American Society for Training and Development Journal. 1960b;18:28-32.

19. Kirkpatrick DL, Kirkpatrick JD. Transferring learning to behavior: Using the four levels to improve performance. San Francisco: Berrett - Koehler Publishers. 2005.

20. Kirkpatrick DL, Kirkpatrick JD. Evaluating training programs: The four levels. 3rd ed. San Francisco: Berrett - Koehler Publishers; 2006.

21. Phillips PP, Phillips JJ, Stone DR, Burkett $H$. The ROI Fieldbook: Strategies for Implementing ROI in HR. Oxford: Routledge - Elsevier; 2007.

22. DeSiltets L. Calculating the Financial Return on Educational Programs. The Journal of Continuing Education in Nursing 2010;41(4):149-50.

23. GeoLearning, Inc. (2003), Training Analytics \& Human Capital ROI, BLM National Training Center (NTC), http://www.ntc.blm.gov/ihp/http://www. pttmedia.com/newmedia_knowhow/KnowHow_ROI/ROI/Analytics_White_ Paper.pdf.

24. Startups.ro. Workshop Essentials of ROI Methodology. [online]. 2009. Available from: URL:www.startups.ro/workshop-essentials-roi-methodology.

25. ROI Institute Romania. ROI in Human Resources Initiatives. The First Case Study in Romania. [online]. 2008. Available from: URL:http://www. comunicatedepresa.ro/united-business-development/roi-institute-romaniafurnizeaza-un-instrument-credibil-pentru-a-masura-aportul-departamentuluide-hr-in-cifra-de-afaceri-a-organizatiei-client/.

26. ROI Institute Inc. ${ }^{\mathrm{TM}}$. Certification in the ROI Methodology. The Complete Impact Measurement Certification System. [online]. 2009. Available from: URL: http://www.roiinstitute.net/wp-content/uploads/2014/10/2013Certification-Workbook.pdf.

27. Moldovan L., Marian L. Competences of Online Teacher Startpoint in the Human Resource Procedure of the Quality Management System. Quality Management in Higher Education. Proceedings of the 4th International Seminar on the Quality Management in Higher Education, Sinaia, Romania, 2006a,127-30.Available from: URL:http://www.isiwebofknowledge.com/
28. Collins C, Buhalis D, Peters M. Enhancing SMTEs' business performance through the Internet and e-learning platforms. Education \& Training. 2003;45:483-94.

29. Haven C, Botterill D. Virtual learning environments in hospitality, leisure, tourism and sport: a review. Journal of Hospitality, Leisure, Sports \& Tourism Education.2003;2(1):75-92.

30. Huang YC, Backman SJ, Chang LL, Backman KF, Mcguire FA. Experiencing student learning and tourism training in a 3D virtual world: an exploratory study. Journal of Hospitality, Leisure, sport \& Tourism Education. 2013;13:190-201.

31. Moldovan L., Marian L. Technological Development for Distance Learning the Requirement of University Quality Management System. Quality Management in Higher Education. Proceedings of the 4th International Seminar on the Quality Management in Higher Education, Sinaia, Romania, 2006b, 359-62. Available from: URL:http://www.isiwebofknowledge.com/

32. Khan BH. Managing E-Learning Strategies: Design, Delivery, Implementation and Evaluation. Hershey: Idea Group Inc.; 2005.

33. Land SM, Hannafin MJ. Patterns of understanding with open-ended learning environments: A qualitative study. Educational Technology Research and Development. 1997;45(2):47-73.

34. Pollard E, Hillage J. Exploring e-learning. Institute of Employment Studies. Report 376; 2001.

35. Afifi GMH. E-learning as an alternative strategy for tourism higher education in Egypt. Quality Assurance in Education 2011;19(4):357-74.

36. Eraqi M, Abou-Alan W, Belal M, Fahmi T. Attitudes of undergraduate students toward e-learning in tourism: the case of Egypt. Journal of Teaching in Travel \& Turism. 2011;11(4):325- 48.

37. Eurlex. The eLearning Action Plan. Designing tomorrow's education. [online]. 2001. Available from: URL: http://eurlex.europa.eu/LexUriServ/LexUriServ. do?uri=COM:2001:0172:FIN:EN:PDF.

38. Sadik A. The readiness of faculty members to develop and implement e-learning: the case of an Egyptian university. International Journal on E-learning2007;6:433-53.

\section{SUMMARY}

- This research aims to measure the impact of some initiative within the human resources over an organization, market leader in the pharmaceutical flied, the measurement of the efficiency concerning the business education programs by human resources e-learning. The research carried-out allow for the application into practice of the theoretical frame of the ROI methodology (Return On Investment) of evaluating the education programs in the human resources business in five stages, suggested by J. J. Phillips. The research validates theoretical data of the model and is focused on the analysis of the gathering process of data required to apply the ROI methodology in the pharmaceutical field. Findings: trainees has perceived a moderate change at organizational level as a result of behavioral changes on the job after training; trainees have perceived as the main benefit for organization quality increase, followed by increase of customer satisfaction, increase in sales, cost cutting, increase of employees satisfaction and increase in productivity; respondents have tried to convert into monetary value the achievements that have been perceived in the reviewed field of activity and have set that approximately half of the estimated value benefits of the organization is due to applying the knowledge and abilities acquired following training. 


\section{About Authors}

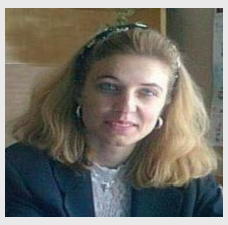

PetruţaBlaga graduated a PhD in Civil Engineering at Technical University of Cluj-Napoca (1997) and a PhD in Management at Faculty of Economic Sciences and Business Administration, "Babeș-Bolyai" University of Cluj-Napoca (2014). She teaches management at "Petru-Maior" University of Tg. Mureş, Faculty of Economics, Juridical and Administrative Sciences, Management-Economic Department; Director of The Education and Training Center of the university. She has published several specialty books (Management of Informational Systems, Operational Management. Optimization of Working Methods, Management in Constructions, etc.), articles in specialty journals and in national and international conference volumes, didactic works. She has attended training and development courses as well as scholarships in Romania and abroad in the field of management, developing and maintaining distance learning, E-learning, enterprise evaluation, etc. She participated in national and international projects and grants.

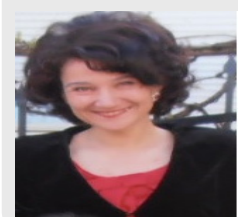

Manuela Rozalia Gabor is associate professor at Petru Maior University of Tirgu Mures (Romania), Management - Economy Department. She has Ph. D. in Cybernetics and Economics Statistic field at University of Economic Studies of Bucharest. She was visiting professor at FH Kufstein (Austria). She has published 6 books, 7 ISI articles, 30 IDB articles, 18 ISI paper proceedings and 11 didactic materials in the field of statistical and econometric methods applied to the market research, tourism, consumer behavior, macro and micro-economic indicators. 